\title{
LAUNAPP - An Android application for Laundry Services
}

\author{
Vinodhini $\mathrm{R}^{1}$, Bhama $\mathrm{S}^{2}$ \\ \{ravivino14@gmail.com¹, sba.mca@psgtech.ac.in² \\ Department of Computer Applications, PSG College of Technology Coimbatore, India ${ }^{1}$, Assistant \\ Professor (SG), Department of Computer Applications, PSG College of Technology Coimbatore, India ${ }^{2}$.
}

\begin{abstract}
Appearance has become a vital part of every person's life, But, life is becoming more complicated with the daily routine work especially for working professionals and students. They do not have spare time to wash their dirty clothes and even do not have enough time to visita laundry shop. This culture has led way to lots of laundry shops that provide service to the people in need. Hence, laundry services have become an integral part of the service industry nowadays. These laundry services can be made accessible to the public with the help of a user-friendly application. Launapp is a great and innovative application that solves the purpose of the people by providing an interface between the service provider and the customer. The full cycle approach of laundry businesses lets clients book and pay for their clothes to be picked up and delivered back clean by tapping several buttons on a smartphone.A mobile application can be built on several platforms, such as Android, and iOS. Android uses Java and iOS uses Swift. But, to create an application for both iOS and Android at the same time can be done using Flutter withsingle codebase. One codebase to maintain, one place to debug, one place to update and one place to rule them all. This is a huge advantage compared to creating apps natively having different places to update and maintain. Flutter requires to know only one language which is Dart.
\end{abstract}

Keywords: Mobile application, flutter, firebase, laundry service, user, admin.

\section{Introduction}

The growing demand for the routine home based services, such as, food, taxi, cleaning and laundryservices have led to the growth industries providing such services. Today, human beings like to spend their valuable time in doing their interested activities, rather than in the household activities [2]. The main aim of building an application for laundry services is to solve one of the greatest problems faced by working professionals as well as others staying out of home getting their clothes washed on time. The demand for application-based laundry services have triggered the customers to register and receive their laundry services with simple clicks. This insisted building a mobile application for laundry services. Also, the need for cross-platform enabled application for providing the services have insisted the use of Flutter. Developers of mobile apps prefer Flutter because the applications developed using Flutter provides a nearnative performance. This paper focuses on establishing a healthy connection between the customers and the laundry service providers to get mutually benefitted, in provisioning and 
availing the on-demand laundry services respectively.

\subsection{Motivations for Laundry App development}

Today's demanding world needs both commodity products and domestic services online. Modern men want things at their place and at their required time, without much complex efforts. As the demand for such simplified availing of services grow within the customer community, the number of providers offering these demanding services also grows rapidly. One such demand is for the laundry-based services, where people like to have these services with few simple taps. Accordingly, the laundry apps are gaining more popularity in the market.

With more research on digital application, there are rare investigations in the laundry sector. Thus, there is a need for redesigning of the business processes that deal with laundry services [3]. At present, the applications that are already existing are unable to allow the customers to track the status of the service. Also, the customers will not able to find if their clothes are missing, as they select only the quantity of clothes. This motivates the development a laundry mobile application to ease the processes involved in managing laundry including record keeping, payments, invoices, scheduling delivery, and pick up of laundry. By embracing this LaunApp mobile application, businesses and customers will be able to effectively deal with the process of managing laundry in a scheduled manner, without any interrupts in the routine activities of the customers.

\subsection{Objectives of Laundry Application}

The proposed system is designed to run as a mobile application, which requires an internet connection, and provides a unified platform for people to get their cleaned clothes. Following are the core objectives of the proposed system:

- Scheduled and door-step servicing through the mobile app.

- Optimize business processes by offering them in the form of a mobile application that handles the activities step by step, allowing a customer to make their own choices.

- Provide a fast and comfortable service for customers by considering the factors like process, performance, and delivery.

\section{Literature Review}

Recent research works have focused on the online services for consumer demands. A review of works done in the development of laundry applications is as follows:

Research works focusing on the application of personality [1] in the development of mobile apps were done. The work included the integration of personality with the demand for the dry-cleaning services and tracing the user experience.

A research article cited the applications of mobile app development technology under a different domain named "Logistics in Life" (LIL) [4]. Its focus is on sectors where traditional logistics are unable to meet the demands of the customers. LIL considers categories such as, searching and exploring locations for storing and transferring goods, tracking orders, picking up and delivery of laundry materials, and so on.

One of the works on laundry app development provided an option for finding the nearest laundry shop for the customers [5]. The key methodology used for this purpose is the data collection related to the location of laundry services at various regional points. 
In order to customize the settings of the preference time for picking up and delivering the clothes and to make paperless transits, Leong and his team [6] has proposed a revised laundry management system.

This paper directs itself towards the development of an easy-to-use mobile application for laundry services, where the customers have a track of the status of their clothes being washed and serviced.

\section{Application Design}

Even though many laundry management applications have been developed, most of the time users are unable to track the status of the service. For example, it may be difficult to find if the clothes are missing because only the quantity of clothes is selected. This is one of the major motivations for the development of LaunApp mobile application to ease the processes involved in managing laundry services, including record keeping, payments, invoices, scheduling delivery, and pick up of laundry. With this LaunApp mobile application, businesses and customers will be able to effectively deal with the process of managing laundry in a scheduled manner that does not cause inconvenience to the customers.

This application seeks to bridge the existing gap by developing reliable mobile application that provides a unified platform with payment options to customers, scheduling pick-up and delivery of laundrythrough an aesthetically pleasing mobile application that will improve the user experience. The main objective is to connect the laundry services and customers by providing quick and comfortable services at the appropriate time.

The process flow of the LaunApp mobile application showing the interaction of the users with the graphical user interface is shown in Figure1. It shows the overall working process of LaunApp application.

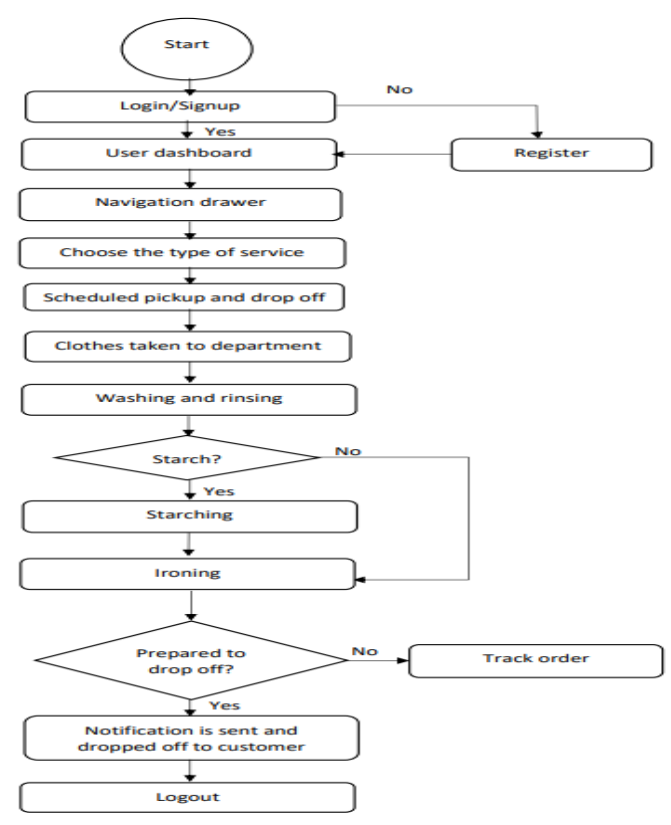

Figure 1: Overall System Design 
As shown, a registered user can login, choose the type of service, add the clothes for laundry, and schedule the pickup details.

The application design for Laundry app includes the following modules:

- Registration module

- Locator module

- Order placement module

- Admin module

\subsection{Registration Module}

Registration module helps the user to register themselves by providing the necessary details. This module is mandatory for every new user who needs to place the order for laundry service. After completing the registration process user can login to the application directly with their email id and password. User can also have the option to reset their password if he/she forgot the password. And this module consists of three pages and are described below:

Signup page - This page helps the new users who are using the application for the first time. And this page is mandatory for new users to get into the application. If the user is already a registered user, then he /she can directly click on login button.

Login page - This page helps the existing users to access the application by providing their login credentials. If the user forgets his/her password, then the user can reset his/her password by clicking forget password button.

Forgot password page - This page allows the users to enter their email id to reset their password.

\subsection{Locator Module}

Locator module allows the user to update their current location and can be able to select the type of laundry service they need for their clothes from the options available. This module consists of two pages,

Location page - This page helps to add the address of the user.

Selecting laundry service - This page helps the user to select the type of service from the list of available options that is displayed.

\subsection{Order Placement Module}

Order placement module helps the user to add the clothes to cart and can schedule timings for picking up the clothes for laundry service they choose. Once the user done with adding clothes to cart, they will able to see the amount to be paid for their service. This module consists of three pages,

Add Clothes - This page helps the user to add the number of specific clothes. Once the quantity of clothes has been selected, the user will be able to see the amount of selected clothes and can confirm the order.

Schedule timings - This page allows the user to select the delivery and pickup timings.

Order Confirmation - This page displays the quantity of clothes and type of service selected with the total amount to be paid. 


\subsection{Admin module}

Admin can be able to register and login to see the orders placed by the customers and can change the status of the service which will reflect in the customer side of tracking status of the service. This module consists of two pages,

Admin Registration - This page allows admins to create their account with email ID and password. The details of the admin will be registered in the database so that while opening the application the admins can login by using their given credentials. Once the details are stored, the admins can login from the Admin Login module.

Admin Login - On opening the application, the admins need to login using their given email ID and password. Once the admin is logged in, the order request will be displayed. In the application, the admin only has the privilege to change the status of work to be displayed.

Any laundry services department has a basic flow of operations. Figure 2 shows the steps involved in the laundry service routine in the form of a sequence diagram.

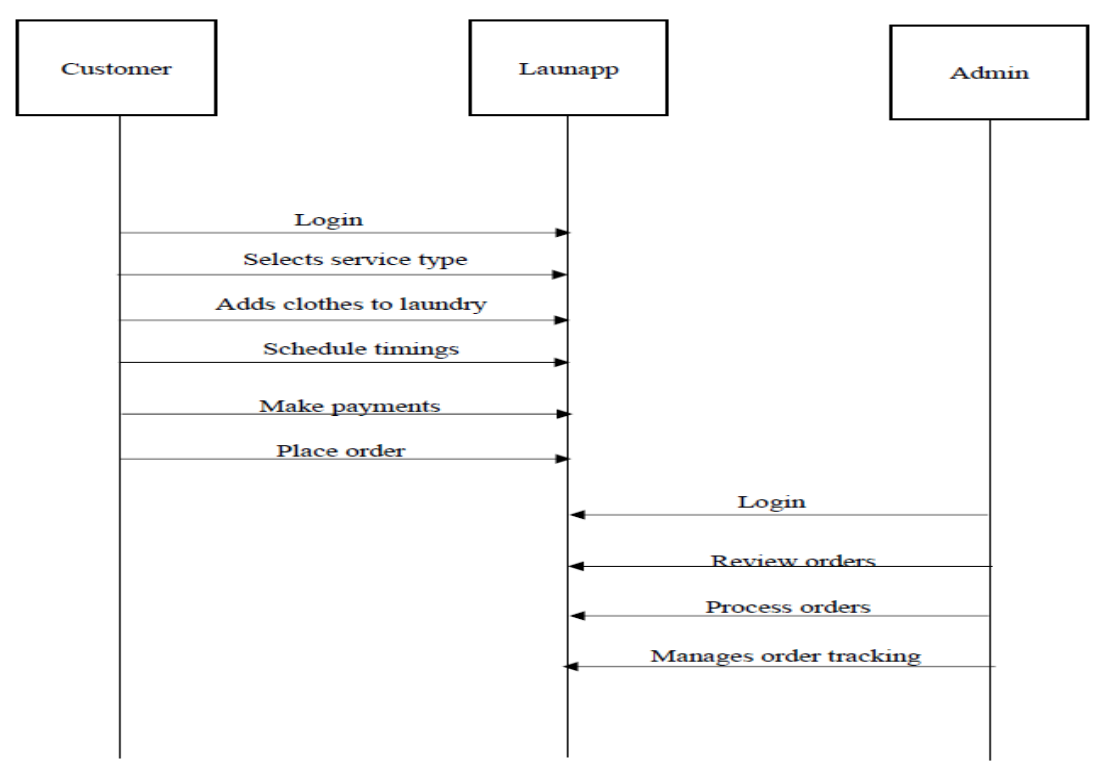

Figure 2: LaunApp - Sequence diagram

As shown, the object interactions arranged in time sequence, which depicts the objects and classes involved in the scenario and the sequence of messages exchanged between the objects. This explains the overall process model of the LaunApp application

\section{Technology Review}

The development of LaunApp mobile application involves the following tools and technologies:

\subsection{Android Studio}


It is an IDE (Integrated Development Environment) for Android operating system. It offers a unique platform to build applications for mobile phones and tablets. Projects can be divided into units that can work independently by structured code modules. Hence, the modules can be built and even tested, and debugged independently. Since there is a built-in support for Google Cloud Platform, integration with Firebase Cloud Messaging is easier.

\subsection{Flutter}

It one of the User Interface toolkits of Google which can be used to create beautiful, and native applications for digital devices, including mobile, desktopand also the web. It also provides a wide variety of readymade widgets that support faster development. It uses Dart language which is easier for developers for effortless reading and visualizing.

\subsection{Firebase}

It is one of the core platforms for app development, especially for the functionalities like data analytics, database management, message passingand report generation. It provides good authentication to build secure systems. It stores data in hierarchical structures, relating the data for easier retrieval.

With such technologies, the LaunApp is built and can be used comfortably.

\section{Case Study}

Customers who wish to get their clothescleanedcan register themselves and login to the application. Once the user logs into the application, user can be able to see the dashboard with the options available for laundry. By clicking the service type, the customer can add clothes to the cart, and at the same time, the amount for the selected clothes will be shown to them.After adding clothes to the cart, the customers will be directed to schedule the timings for picking up of the clothes. And at last,the customer can select to pay the amount either using online payment or cash on delivery option. Once the laundry service is done with, the customer will receive a notification message.

The admin is the one who receives the order request and direct the right person to the customers' location to pickup the clothes for laundry.

Following are the screenshots of the LaunApp mobile application:

Welcome Screen is the first screen where the customer and the admin try to open the application. This screen helps to get into the application by clicking the 'Get started' button at the bottom of the screen. Figure 3 shows the welcome screen of LaunApp.Figure 4 shows the login and signup screen for the user. Users can directly login to the application, if they are already registered. Otherwise, they must register by providing the required details. 


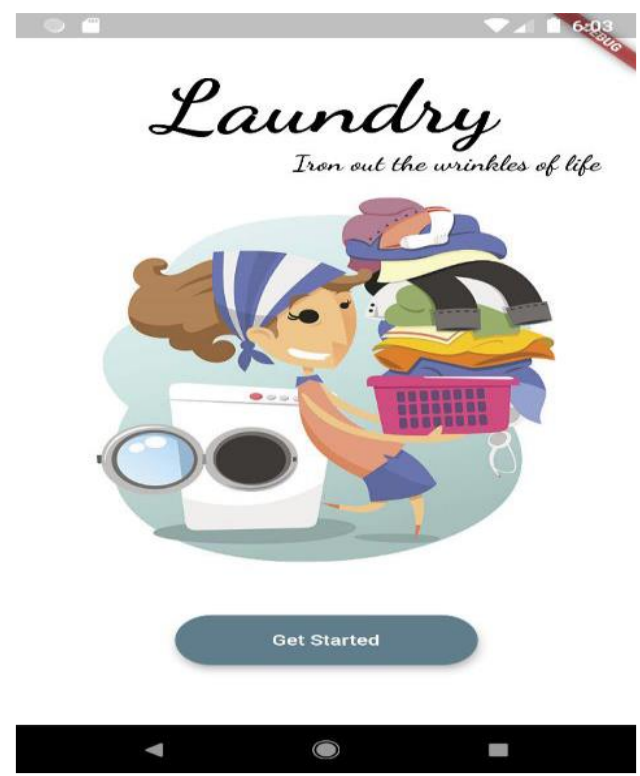

Figure 3: Welcome Screen

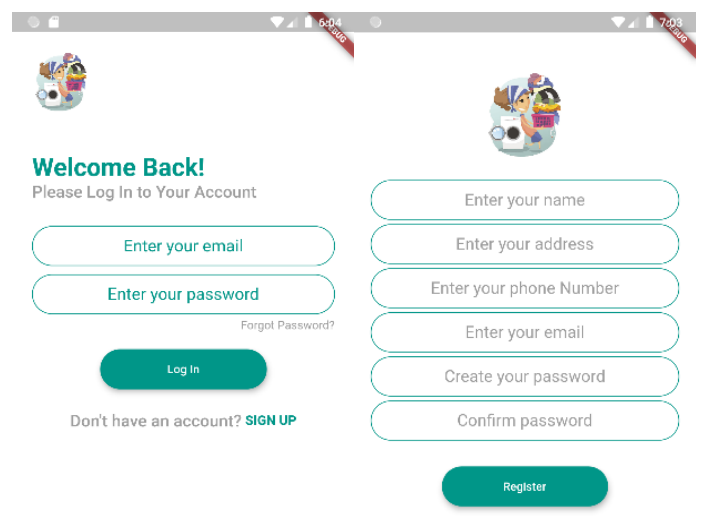

4040

Figure 4: Login and Signup 
Figure 5 shows the types of laundry services available and adding clothes to the cart. This will allow the user to select the type of service and clothes to get their laundry delivered.

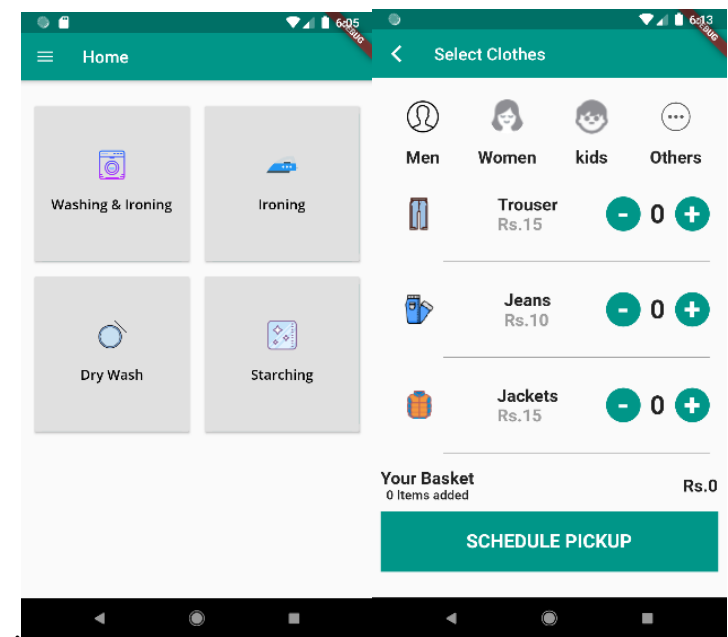

Figure 5: Select Service Type and Add to Cart

Figure 6 allows the user to schedule a pickup date and timings and also allows the user to pay the amount online.

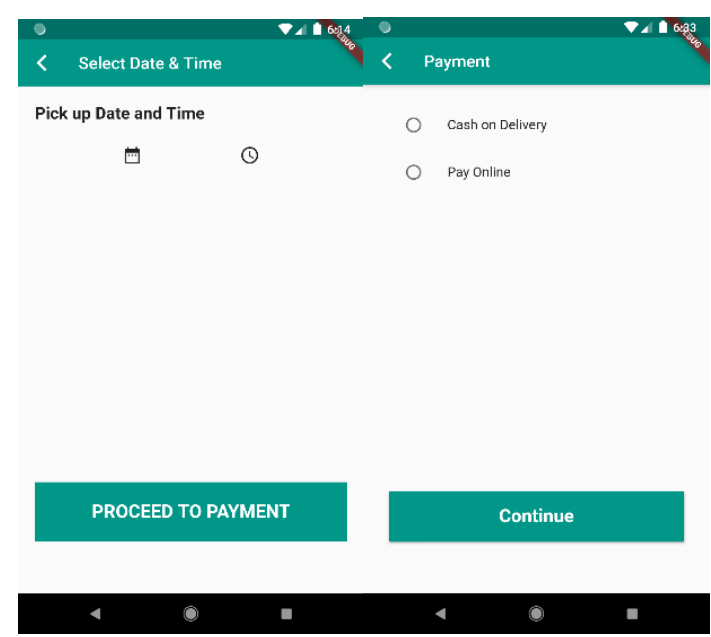

Figure 6: Schedule Timings and Payment

Figure 7 shows the Update location page which allows the user to update the current location in the Google maps. 


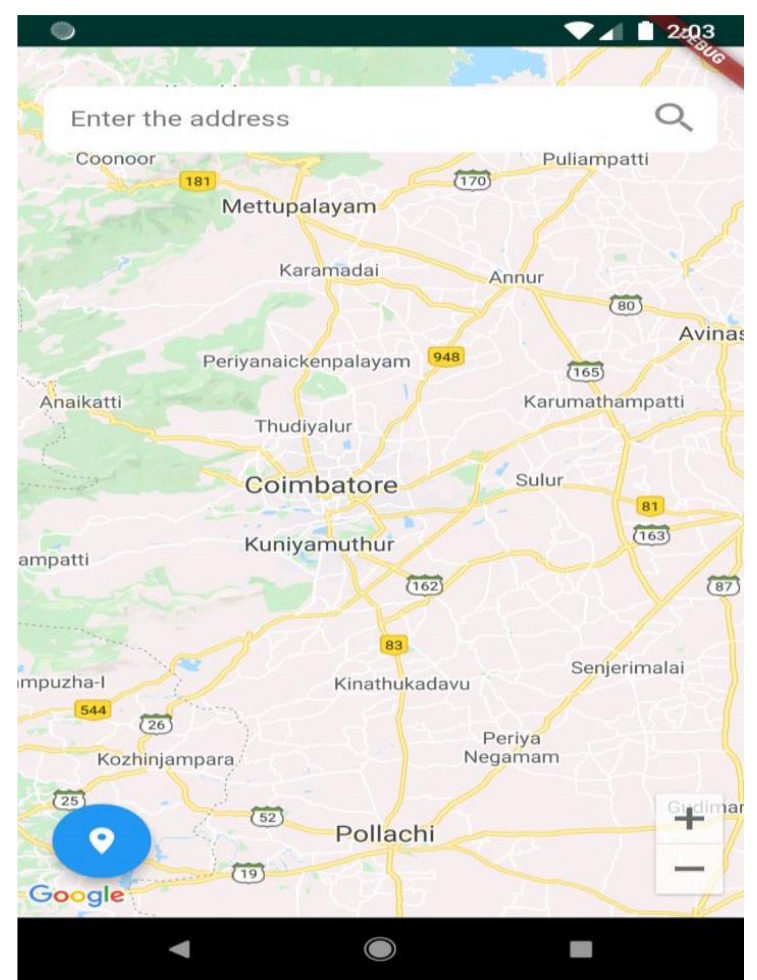

Figure 7: Update Location

User is redirected to this page with the maps integrated to the application when the user wants to update his/ her current location.

\section{Conclusion}

This paper has highlighted the design and development of a mobile app which meets the requirements of individuals regarding technological advancements and innovations in the laundry sector. This laundry service application is developed by using an interactive interface so that both the admin and the customer can directly use this application easily. The system helps users in completingtheir laundry-related services effortlessly, quickly, andefficiently.

The LaunApp application may be enhanced with the following approaches:

- The mobile application may also be converted into a web application.

- Driver module can also be included for picking up the clothes from the user's destination to laundry department and also live tracking can be done for driver module to know the current location of the driver. 


\section{References}

[1] Felix Hedlund, "Development of a Mobile Customer Application for Dry Cleaning Services", Master's Thesis, Department of Design Sciences Lund University, 2015.

[2] Akanksha Gupta, Debendra Kumar Panda, Mayank Pande, "Development of Mobile Application for Laundry Services Using Android Studio ", International Journal of Applied Engineering Research, ISSN 0973-4562 Volume 13, Number 12, pp: 10623-10626, 2018.

[3] Doaa M. Bamasoud*, Asma M. Alqahtani, Eman A. Aljdea, Reem A. Alshomrani, Maha S. Alshahrani, Zohoor A. Alghamdi, Ameerah M. Alghamdi, Shahd F. Almaawi, Asrar D. Alshahrani, "An Explorative Study for Laundry Mobile Application Laundry Process Change", (IJACSA) International Journal of Advanced Computer Science and Applications, Vol. 9, No. 4, pp: 96-99, 2018.

[4] Dae-Ho Byun, Han-Na Yang and Dong-Seop Chung, "Evaluation of Mobile Applications Usability of Logistics in Life Startups", Sustainability 2020, 12, 9023, October 2020.

[5] Ivan Theomanto, Fikri Fadillah Fahmi, Machfito Imanu Yuhyi, Alvina Aulia, "The Development of Android-Based Mobile Application of LaundryPlaces", Journal of Critical Reviews", Vol 7, Issue 08, 2020

[6] Leong Yi Mei, Ku Nurul Fazira Ku Azir, Siti Zuraidah Ibrahim and Saidatul Norlyana Azemi, "LaundryMama: Humanising Laundry Tasks using Laundry Management System and LaundryOnDemand Mobile Applications", IOP Conference Series: Materials Science and Engineering, Volume 767, 1st International Symposium on Engineering and Technology (ISETech), 2020. 